\title{
Research Progress of Anti-PD-1/PD- L1 Immunotherapy Related Mechanisms and Predictive Biomarkers in NSCLC
}

\author{
Fenglong Bie ${ }^{1,2}$, He Tian ${ }^{1,2}$, Nan Sun ${ }^{1,2}$, Ruochuan Zang ${ }^{1}$, Moyan Zhang ${ }^{1}$, Peng Song ${ }^{1}$, \\ Lei Liu ${ }^{1}$, Yue Peng ${ }^{1}$, Guangyu Bai ${ }^{1}$, Bolun Zhou ${ }^{1}$ and Shugeng Gao ${ }^{1 *}$ \\ 1 Department of Thoracic Surgery, National Cancer Center/National Clinical Research Center for Cancer/Cancer Hospital, \\ Chinese Academy of Medical Sciences and Peking Union Medical College, Beijing, China, ${ }^{2}$ State Key Laboratory of \\ Molecular Oncology, National Cancer Center/National Clinical Research Center for Cancer/Cancer Hospital, Chinese \\ Academy of Medical Sciences and Peking Union Medical College, Beijing, China
}

Programmed cell death-1 (PD-1)/programmed death-ligand 1 (PD-L1) is an important pair of immune checkpoints $(\mathrm{IC})$, which play an essential role in the immune escaping process of tumors. Anti-PD-1/PD-L1 immunotherapy can block the suppression effect of the immune system produced by tumor cells through the PD-1/PD-L1 axis and restore the pernicious effect of the immune system on tumor cells. The specific mechanism of antiPD-1/PD-L1 immunotherapy is closely related to PI3K (phosphatidylinositol 3-kinase)/ AKT (AKT serine/threonine kinase 1), JNK (c-Jun N-terminal kinase), NF-kB (nuclear factor-kappa B subunit 1), and other complex signaling pathways. Patients receiving antiPD-1/PD-L1 immunotherapy are prone to drug resistance. The mechanisms of drug resistance mainly include weakening recognition of tumor antigens by immune cells, inhibiting activation of immune cells, and promoting the production of suppressive immune cells and molecules. Anti-PD-1/PD-L1 immunotherapy plays a vital role in nonsmall cell lung cancer (NSCLC). It is essential to find better efficacy prediction-related biomarkers and screen patients suitable for immunotherapy. At present, common biomarkers related to predicting immune efficacy mainly include PD-L1 expression level in tumors, tumor mutation burden (TMB), microsatellite instability (MSI)/mismatch repair (MMR), mutations of driver gene, etc. However, the screening efficacy of each indicator is not ideal, and the combined application of multiple indicators is currently used. This article comprehensively reviews anti-PD-1/PD-L1 immunotherapy-related mechanisms, drug resistance-related mechanisms, and therapeutic efficacy-related predictive biomarkers.

Keywords: non-small cell lung cancer (NSCLC), programmed cell death-1 (PD-1)/programmed death-ligand 1 (PD-L1), immunotherapy, predictive biomarkers, drug resistance

\section{INTRODUCTION}

Lung cancer is the tumor with the second morbidity rate and the first mortality rate globally $(1,2)$. Nonsmall cell lung cancer (NSCLC) accounts for $85 \%$ of lung cancer (3). At present, the main treatments for NSCLC are surgery, radiotherapy, chemotherapy, and targeted therapy (3-5). With the rise of immunotherapy in recent years, immunotherapy has also become one of the main treatments for 
NSCLC (6). Programmed cell death-1 (PD-1, also called CD279)/ programmed death-ligand 1 (PD-L1, also known as CD274) is a pair of critical immune checkpoints (IC) and tumor cells can inhibit the killing effect of the immune system by activating the PD-1/PD-L1 axis (7). The anti-PD-1/PD-L1 immunotherapy can block the PD1/PD-L1 axis and restore the lethal effect of the immune system on tumor cells (8). After binding of PD-1 to PD-L1, it can antagonize T cell (antigen) receptor (TCR) recognition by phosphorylating the Src homology 2 domain tyrosine phosphatases (SHP-2) site and block T cells from functioning (9). The activity of T cells is inhibited, leading to immune escaping of tumor cells through phosphatidylinositol 3-kinase (PI3K)/AKT serine/threonine kinase 1 (AKT)/mammalian target of rapamycin (mTOR) and other signaling pathways (10).

Patients receiving immunotherapy often develop drug resistance. Current studies have shown that drug resistance occurs mainly through weakening recognition of tumor antigens by immune cells, inhibiting activation of immune cells, and promoting the production of suppressive immune cells and molecules $(11,12)$. The current common prediction markers of immunotherapy effect include PD-L1 expression level of tumor tissue, tumor mutation burden (TMB), microsatellite instability (MSI)/mismatch repair (MMR), related driver gene mutations, etc. But the predictive effect of each marker is not ideal, and a combination of multiple indicators is usually used (13-15).

Given current research on anti-PD-1/PD-L1 immunotherapy, we have found some problems. Some patients have higher PD-L1 expression, but the therapeutic effect is not ideal. On the contrary, some patients have lower or even negative PD-L1 expression, but the therapeutic effect is well. Biomarkers such as TMB, Tumor Proportion Score (TPS), MSI/MMR, driver gene mutations are not perfect for evaluating the efficacy of immunotherapy. At present, there is no ideal predictive biomarker for the therapeutic effect of immunotherapy, and a combination of multiple indicators is usually used to improve the predictive efficiency. According to reports, the current effective rate of immunotherapy in lung cancer is not more than $30 \%$ (16), and the overall effective rate is just about $20 \%$ (17). Therefore, it is essential to find suitable biomarkers for immune efficacy prediction and screen out appropriate patients for immunotherapy. Based on the above questions, we want to explore anti-PD-1/PD-L1 immunotherapy efficacy and drug resistance related mechanisms in NSCLC. At the same time, we hope to find more reliable biomarkers for predicting immunotherapy efficacy. We have made a comprehensive review of these issues.

\section{MECHANISMS RELATED TO THE EFFICACY OF ANTI-PD-1/PD-L1 IMMUNOTHERAPY}

\section{Structure and Expression of PD-1 and PD-L1}

The IC proteins currently discovered include PD-1, PD-L1, cytotoxic T lymphocyte-associated molecule-4 (CTLA-4), lymphocyte-activation gene 3 (LAG3), T cell immunoglobulin, mucin domain-containing protein 3 (TIM3), etc. But the most researched ICs are PD-1 and PD-L1 $(18,19)$. PD-1 is a transmembrane protein encoded by human programmed cell death protein 1 (PDCD1) $(20,21)$, which is mainly expressed on the surface of activated T cells, B cells, and natural killer (NK) cells (22). PD-1 can exert an immunosuppressive effect by combining PD-L1/programmed cell death 1 ligand 2 (PD-L2, CD273) (23). Both PD-L1 and PD-L2 belong to the B7 protein family (24). PD-L1 is mainly expressed on the surface of tumor cells, immune cells, epithelial cells, and endothelial cells. In contrast, PD-L2 is primarily expressed on the surface of dendritic cells and macrophages (25). PD-L1 is more widely expressed on normal cells and tumor cells, and its role is much more significant than that of PD-L2, so most of the current studies focus on PD-L1 (26).

Many studies focus on the protein expression level of PD-L1 in tumor cells. Most of these studies have shown that the level of $\mathrm{PD}-\mathrm{L} 1$ expression is closely related to the efficacy of anti-PD-1/ PD-L1 immunotherapy $(27,28)$. The commonly used parameter in NSCLC to reflect the expression level of PD-L1 is the TPS score, which is the percentage of positive tumor cells. TPS $=(\mathrm{PD}$ L1 staining positive tumor cells)/(total live tumor cells) ${ }^{\star} 100 \%$ (29). PD-L1 expression levels can be divided into two types, innate and adaptive immune expression. The expression mode of innate immunity is regulated by genes and has a certain fixity (19). The expression mode of adaptive immunity is affected by immune and inflammatory factors, such as interleukin, interferon, tumor necrosis factor, etc. This expression mode will change with the dynamic changes of the tumor immune microenvironment (19).

\section{Mechanisms of PD-1/PD-L1 Inhibitors}

The tumor immunoediting processes are divided into three steps: immune elimination, immune balance, and immune escape (12, 30). PD-1/PD-L1 are essential immune checkpoints, which can act as a brake on the immune system and play a crucial role in the immune escape process of tumors (31). A combination of PD-1 and PD-L1 can generate immunosuppressive signals and inhibit the follow-up effects of the immune system (32). PD-L1 molecules are highly expressed on the surface of tumor cells. PD-L1 binds to PD-1 molecules on the surface of immune cells (mainly T lymphocytes), which can induce immune cell exhaustion and inhibit the direct killing effect of immune cells on tumors. It can induce immune cells (primarily $\mathrm{T}$ helper cells) to secrete immunosuppressive factors to inhibit further the immune system's killing effect on tumor cells (33).

After PD-1 binds to PD-L1, it will lead to the phosphorylation of the immunoreceptor tyrosine inhibitory motif (ITIM) and immunoreceptor tyrosine switching motif (TISM) of PD-1, and then inhibit $\mathrm{T}$ cell activation by recruitment of protein tyrosine phosphatase Src homology 1 domain tyrosine phosphatases (SHP-1) and SHP-2, or by up-regulating the expression of ATF-like alkaline leucine zipper transcription factor (BATF) (34). PD-1/PD-L1 immune checkpoint inhibitors (ICIs), by binding to PD-1 or PD-L1, block the binding of PD-L1 on the surface of tumor cells and PD-1 on the surface of immune cells, as shown in Figure 1, 


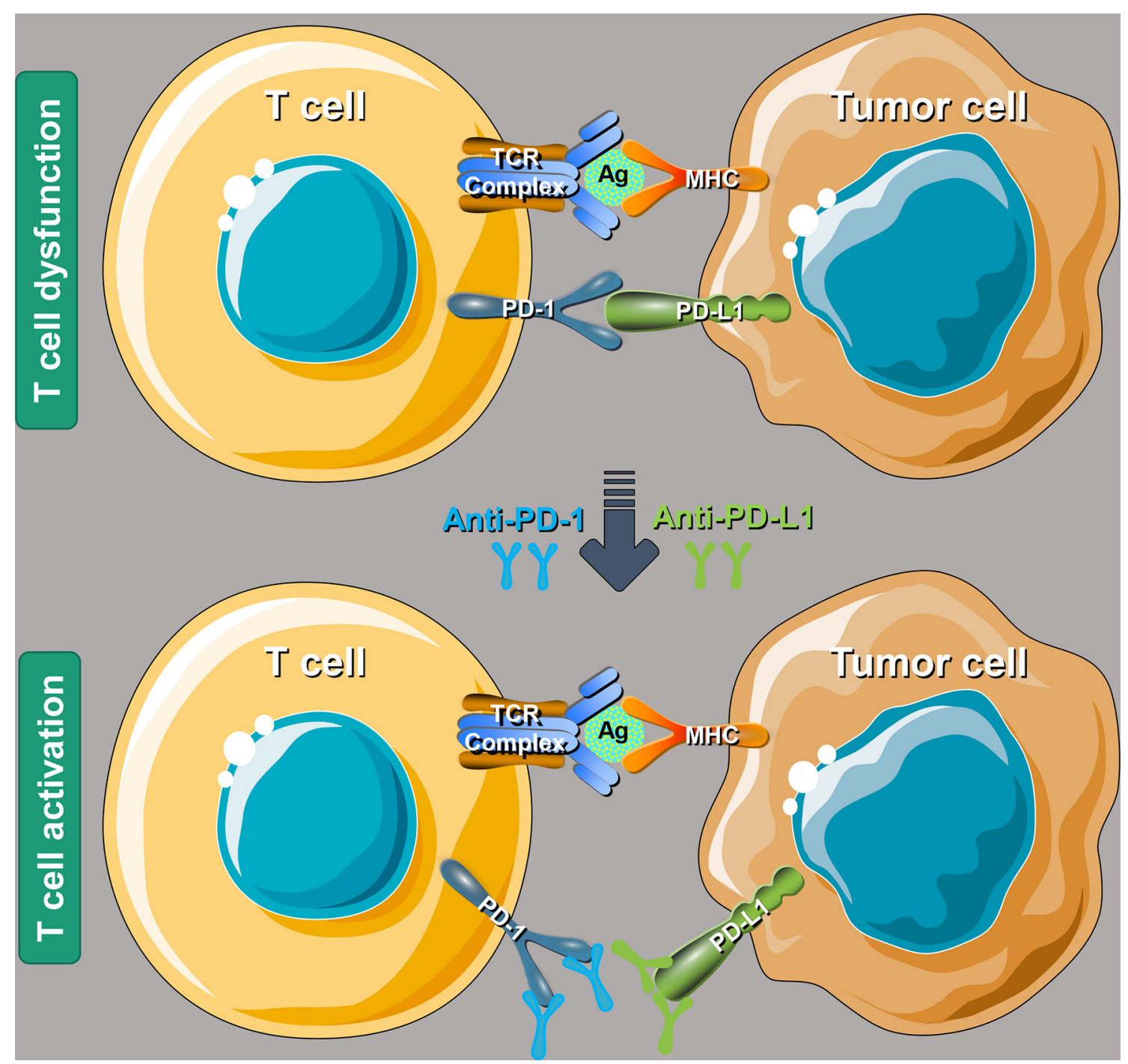

FIGURE 1 | A schematic diagram of the molecular mechanism using PD-1/PD-L1 ICls to rescue T cell functions. PD-1, programmed cell death-1; PD-L1, programmed death-ligand 1; ICls, immune checkpoint inhibitors; TCR, T cell receptor; Ag, antigen; MHC, major histocompatibility complex.

to relieve the inhibitory effect of tumor cells on the immune system and restore killing effect of the immune system on tumor cells $(35,36)$.

\section{DRUG RESISTANCE-RELATED \\ MECHANISMS OF ANTI-PD-1/PD-L1 IMMUNOTHERAPY}

\section{Attenuate Recognition of Tumor Antigens by Immune Cells}

The frequency of gene mutations in tumor cells is high, which can cause abnormal expression of related molecules for processing and presenting tumor antigens, such as the downregulation of major histocompatibility complex (MHC)-I molecule expression. Abnormal expression of these molecules will cause immune cells to fail to recognize tumor cells usually, thereby mediating the immune tolerance of tumor-associated antigens, resulting in immune cells not being able to effectively identify and kill tumor cells $(37,38)$.

\section{Inhibit Activation of Immune Cells}

Tumor cells can release various immunosuppressive factors, such as adenylate, indoleamine 2,3-dioxygenase 1 (IDO), prostaglandin E2 (PEG2), interleukin-10 (IL-10), and transforming growth factor- $\beta$ (TGF- $\beta$ ), to inhibit the activation of immune cells (39). At the same time, tumor cells can also promote the expression of immunosuppressive molecules, such as PD-1, PD-L1, PD-L2, CTLA-4, and further inhibit the activation of immune cells (40). 


\section{Promote Production of Suppressive Immune Cells and Molecules}

Tumor cells can induce the production of immunosuppressive cells, such as T regulatory cells, natural killer T (NKT) cells, and bone marrow-derived immunosuppressive cells $(41,42)$. Immune cells can play an immunosuppressive role by contacting other cells and releasing corresponding inhibitory immunoregulatory factors. By up-regulating molecules related to the signal axis, such as PDL1/PD-1, Fas ligand (FASL)/FAS, inhibitory regulatory factors can mediate the depletion of T cells (37).

\section{Affect Tumor Stromal Cells and Change TME}

In tumor microenvironment (TME), tumor cells can promote the proliferation and aggregation of fibroblasts, macrophages, bone marrow-derived immunosuppressive cells, $\mathrm{T}$ regulatory cells, and NKT cells, and change tumors by contacting each other or secreting cytokines to inhibit the normal function of the immune system in killing tumor cells. TME may weaken the ability of the immune system to recognize and kill tumor cells $(42,43)$.

\section{IMMUNOTHERAPY EFFICACY PREDICTION RELATED BIOMARKERS}

Different NSCLC patients acquire different efficacy of anti-PD-1/ PD-L1 immunotherapy due to individual differences. It is essential to find suitable biomarkers for predicting efficacy and screen out patients ideal for immunotherapy. At present, the commonly used clinical and immunological efficacy prediction biomarkers mainly include PD-L1 expression, TMB, microsatellite instability-high (MSI-H)/deficient mismatch repair (dMMR), etc. However, each has its corresponding shortcomings, so a combination of several biomarkers is usually used to improve the efficacy of predicting. This article focuses on the following predictive biomarkers related to antiPD-1/PD-L1 immunotherapy (as shown in Table 1 and Figure 2): PD-L1, TMB, MSI-H/dMMR, tumor DNA-related biomarkers, peripheral blood-related biomarkers, intestinal flora-related biomarkers, TME-related biomarkers, T cellrelated biomarkers, and immunohistochemistry (IHC) related biomarkers.

\section{PD-L1 Expression Level}

The expression level of PD-L1 in tumor tissues of patients is one of the most important biomarkers for whether patients choose ICIs therapy. At present, most studies have shown that NSCLC patients with high PD-L1 expression have a better therapeutic effect receiving anti-PD-1/PD-L1 immune treatment $(44,45)$. The commonly used indicator of PD-L1 expression level in NSCLC is TPS, TPS $=(\mathrm{PD}-\mathrm{L} 1$ staining positive tumor cells $) /$ (total live tumor cells) ${ }^{\star} 100 \%$ (29). However, there is still controversy about the predictive efficacy of tumor PD-L1 expression. Studies have shown that the expression of PD-L1

TABLE 1 | Summary of predictive biomarkers using PD-1/PD-L1 ICls in NSCLC.

\begin{tabular}{|c|c|c|c|}
\hline Category & Sub-Category & Biomarker & Example \\
\hline \multirow[t]{14}{*}{ Tumor } & DNA Biomarkers & $\mathrm{dMMR/MSI-H}$ & - \\
\hline & & $\mathrm{TMB}$ & - \\
\hline & & DNA repair genes & POLD1, POLE, MSH2 \\
\hline & & Other genes & STK11, MHC, B2M, EGFR \\
\hline & Protein Biomarkers & PD-L1 & - \\
\hline & & Tumor neoantigens & - \\
\hline & & Other immune checkpoints & CTLA-4, LAG3, TIM3 \\
\hline & TME related Factors & Immune cells infiltration & CD4+ T cells, CD8+ T cells \\
\hline & & Cytokines or chemokines & TGF, TNF, interleukin \\
\hline & & Stromal composition & Cancer-associated fibroblast \\
\hline & T cell Biomarkers & Effector T cell & CD4+ T cells, CD8+ T cells \\
\hline & & T cell inflamed GEP & CCL5, CXCL13 \\
\hline & & TILs & CD8+ T cells, NK cells \\
\hline & & TCR sequencing & CDR3 \\
\hline \multirow[t]{6}{*}{ Blood } & DNA Biomarkers & bTMB & - \\
\hline & & cfDNA & SNV, fragment, CNV \\
\hline & Cell Biomarkers & Flow cytometry cell immunophenotyping & CD4+ T cells, CD8+ T cells \\
\hline & & Flow cytometry TCR immunophenotyping & CDR3 \\
\hline & Other Blood Biomarkers & Exosomal PD-L1 & - \\
\hline & & Cytokines & TGF, TNF, interleukin \\
\hline \multirow[t]{3}{*}{ Gut Microbiota } & & Bacteroides & - \\
\hline & & Bifidobacterium & - \\
\hline & & Akkermansia muciniphila & - \\
\hline
\end{tabular}

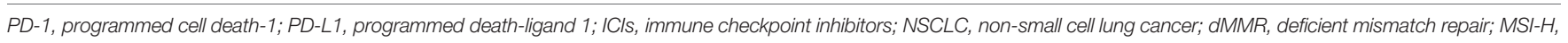

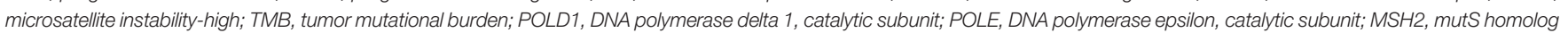

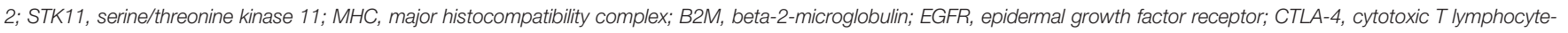

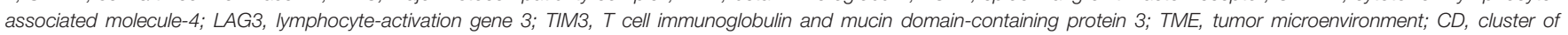

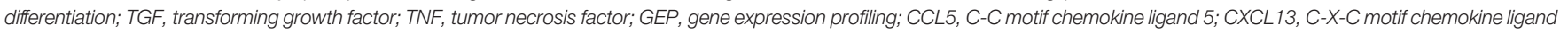
13; TILs, tumor-infiltrating lymphocytes; NK cell, natural killer cell; TCR, T cell receptor; CDR3, complementarity determining region 3; bTMB, blood tumor mutational burden; cfDNA, circulating-free DNA; SNV, single nucleotide variant; CNV, copy number variation. 


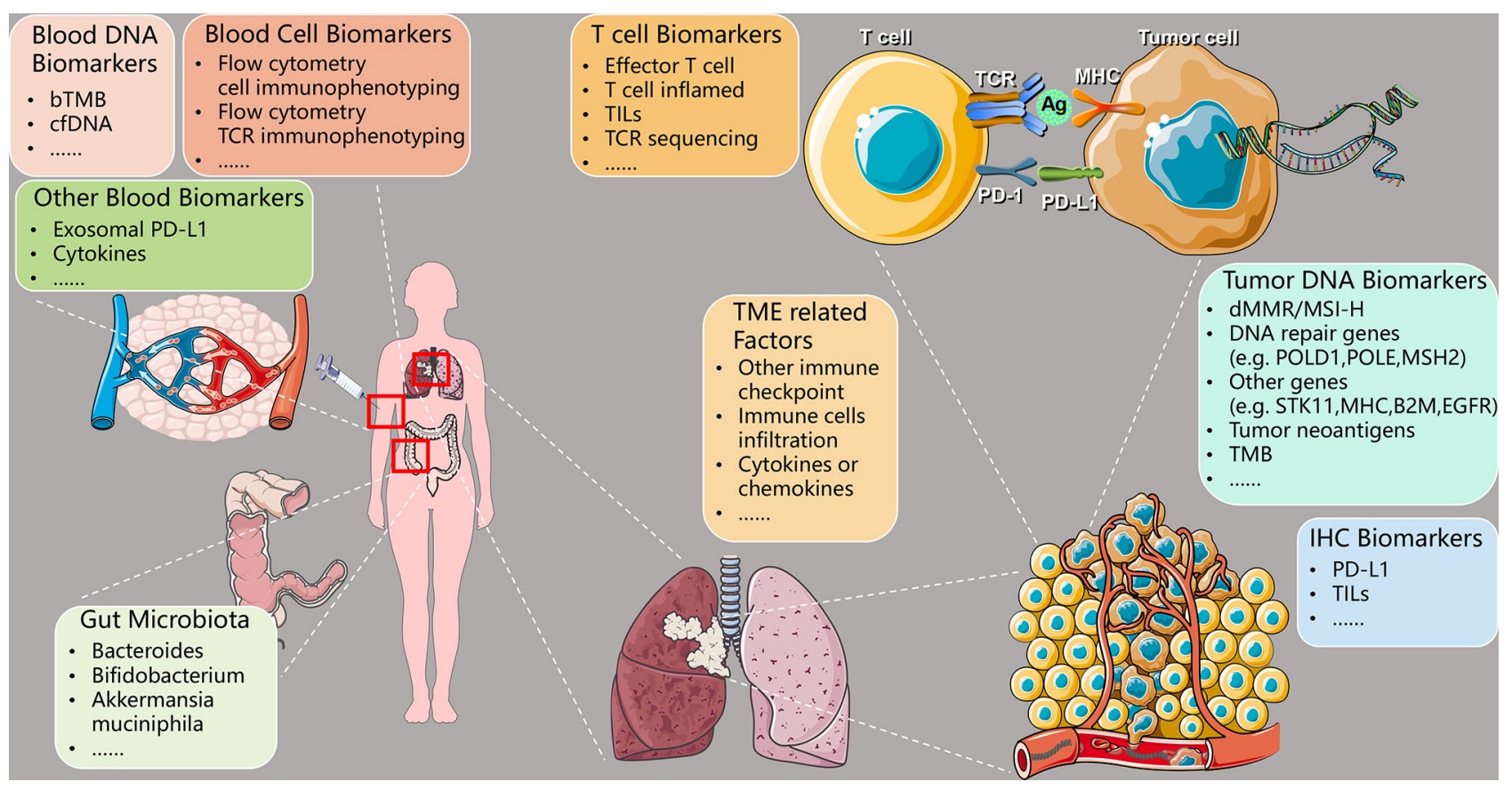

FIGURE 2 | Predictive biomarkers of therapeutic efficacy using PD-1/PD-L1 ICls in NSCLC. PD-1, programmed cell death-1; PD-L1, programmed death-ligand 1; ICls, immune checkpoint inhibitors; NSCLC, non-small cell lung cancer; TCR, T cell receptor; Ag, antigen; MHC, major histocompatibility complex; bTMB, bloodbased tumor mutational burden; cfDNA, circulating-free DNA; TILs, tumor-infiltrating lymphocytes; TME, tumor microenvironment; dMMR, deficient mismatch repair; MSI-H, microsatellite instability-high; POLD1, DNA polymerase delta 1, catalytic subunit; POLE, DNA polymerase epsilon, catalytic subunit; MSH2, mutS homolog 2 STK11, serine/threonine kinase 11; B2M, beta-2-microglobulin; EGFR, epidermal growth factor receptor; TMB, tumor mutational burden; IHC, immunohistochemistry.

in some NSCLC patients is low or even negative, but the effect of anti-PD-1/PD-L1 treatment is better (46). On the contrary, some patients have higher expression of $\mathrm{PD}-\mathrm{L} 1$, and the therapeutic effect of anti-PD-1/PD-L1 therapy is poor.

\section{TMB}

TMB has an excellent predictive value for the efficacy of immunotherapy. Studies have shown that TMB is an independent prognostic factor related to the effectiveness of immunotherapy and is not affected by the expression level of other indicators (such as PD-L1) (14). At present, many studies have shown patients with high TMB in tumors have a better therapeutic effect on anti-PD-1/PD-L1 immunotherapy $(47,48)$, and the same is true for NSCLC patients (49). Detection of TMB in the blood (bTMB) is also a new TMB detection method. Studies have shown that bTMB is related to the immune therapeutic efficacy of NSCLC (50), but more studies are still needed to verify it. Although both the expression level of PD-L1 and $\mathrm{TMB}$ are related to the efficacy of patients receiving immunotherapy, studies have shown that there is no exact correlation between PD-L1 expression and TMB (51).

\section{MSI-H/dMMR}

Current studies have shown that MSI-H/dMMR can predict the efficacy of immunotherapy for gastric cancer and colon cancer, but the incidence of MSI-H/dMMR in lung cancer is low (52).
Therefore, further research needs whether MSI-H/dMMR can be used as a predictive biomarker for NSCLC immunotherapy. At present, the standard measure commonly used to judge MSI-H is the Bethesda method (53). Research by Vanderwalde et al. showed that patients with MSI-H have a higher probability of having high TMB, but not vice versa (54).

\section{Tumor DNA-Related Biomarkers}

In addition to classic prediction biomarkers of immune efficacy, such as TMB and MSI-H/dMMR described above, studies have shown that the existence of specific driver gene mutations is related to the effectiveness of immunotherapy. For example, the typically favorable genes in NSCLC are TP53 (tumor protein p53), KRAS (KRAS proto-oncogene, GTPase), etc. Common negative genes include EGFR (epidermal growth factor receptor), $M E T$ (MET proto-oncogene, receptor tyrosine kinase), $A L K$ (ALK receptor tyrosine kinase), etc. Patients with favorable gene mutations will have better therapeutic effects when receiving immunotherapy, while patients with negative gene mutations will have poorer efficacy. But a larger cohort is still needed for verification $(19,55)$. It has been reported that patients with driver gene mutations usually have lower TMB, while patients with high TMB usually have negative driver genes (56). Research by Garassino et al. showed that, regardless of EGFR/ALK mutation status, Durvalumab (anti-PD-L1 
monoclonal antibody) has an excellent therapeutic effect for patients with greater or equal to $25 \%$ PD-L1 expression (57).

Tumor cells are prone to generate new mutations due to genome instability. The tumor-specific antigens produced by new mutations are called tumor neoantigens (58). Current studies have shown that tumor neoantigen is highly immunogenic and can activate CD4+ and CD8+ T cells to produce an immune response, which is expected to become a predictive biomarker in cancer immunotherapy (59).

\section{Peripheral Blood-Related Biomarkers}

We divide peripheral blood-related immunotherapy efficacy biomarkers into three categories: blood DNA-related biomarkers, blood cell-related biomarkers, and other blood-related biomarkers.

Blood DNA-related biomarkers mainly include bTMB and circulating-free DNA (cfDNA). Wang Z et al. mainly used NextGeneration Sequencing technology and optimized algorithms to explore the relationship between bTMB and TMB in tumor tissue, indicating that bTMB can be used as an efficacy prediction biomarker of anti-PD-1/PD-L1 immunotherapy in NSCLC (60). The Brazos-Vázquez EM et al. team reviewed the application of liquid biopsy in immunotherapy of NSCLC patients, including circulating tumor cells (CTCs), cfDNA, and exosomes. It showed that liquid biopsy tools are expected to become promising predictive biomarkers for immunotherapy (61). Giroux Leprieur E et al. performed whole-exome sequencing on ctDNA to calculate bTMB and identify critical features, such as single nucleotide variants (SNVs), copy number variations (CNVs), to predict the efficacy of ICIs on advanced lung adenocarcinoma (62). Nabet BY et al. demonstrated that ctDNA and peripheral CD8+ T cell levels ahead of ICIs treatment are independently associated with durable clinical benefit for NSCLC patients receiving ICIs (63).

Blood cell-related biomarkers mainly include immune cell and $\mathrm{T}$ cell receptor (TCR) immunophenotyping by flow cytometry. The immune cells in peripheral blood can be classified through flow cytometry, and their number can be calculated (64). The type and number of immune cells (65), and TCR immunophenotyping (66), are both closely related to the efficacy of anti-PD-1/PD-L1 immunotherapy. Fumet JD et al. evaluated the role of CD8 under anti-PD-1 therapy and demonstrated that CD8 was a promising prognostic and predictive factor in NSCLC (67). The study result of Gettinger SN et al. showed CD3+ TILs related signal was associated with favorable response to ICIs therapy in NSCLC (68). The research of Anagnostou V et al. indicated that TCR clonal expansions within the tumor tissue or in circulating $\mathrm{T}$ cells were critical indicators to the therapeutic response of ICIs in NSCLC (69).

Other blood-related biomarkers mainly include exosomal PD$\mathrm{L} 1$ and cytokines. Xie F et al. showed that exosomal PD-L1 plays a vital role in developing drug resistance in immunotherapy (70). Exosomal PD-L1 may potentially become a target for overcoming resistance to anti-PD-1/PD-L1 therapy (70). Current studies have shown that circulating exosomes play an essential role in developing tumors and the immune process of anti-tumor and have a good efficacy predictive value of patients receiving ICIs (71, 72). Interferon-gamma (IFN- $\gamma$ ), tumor necrosis factor (TNF), and other cytokines also play an essential role in the process of antiPD-1/PD-L1 immunotherapy (73). All the above results indicate that peripheral blood-related biomarkers are promising indicators for predicting the therapeutic effect of ICIs.

\section{Biomarkers Related to Intestinal Flora}

The predictive biomarkers related to intestinal flora of immunotherapy efficacy mainly include Bacteroides, Bifidobacterium, Akkermansia muciniphila. Vétizou $\mathrm{M}$ et al. showed that the anti-tumor effects of CTLA-4 blockers depend on the subclassification of Bacteroides species (74). The gut microbiome determines the different degrees to which ICIs can elicit the anti-cancer immune response (75). Current studies have shown that the type and quantity of intestinal microbes are closely related to the therapeutic effect of receiving ICIs.

\section{TME-Related Biomarkers}

TME-related biomarkers mainly include other kinds of immune checkpoints, immune cells infiltration, and cytokines or chemokines. In addition to PD-1/PD-L1 ICs discussed explicitly in this article, common ICs include CTLA-4, LAG3, TIM3 (hepatitis A virus cellular receptor 2), and TIGIT (T cell immunoreceptor with Ig and ITIM domains), which also play essential roles in the process of anti-PD-1/PD-L1 immunotherapy $(76,77)$. The composition of immune cells in TME, such as CD8+ $\mathrm{T}$ cell, $\mathrm{T}$ regulatory cell, and the ingredient of cytokines and chemokines, are also closely related to the efficacy of anti-PD-1/ PD-L1 immunotherapy $(78,79)$.

\section{T Cell-Related Biomarkers}

$\mathrm{T}$ cell-related efficacy prediction biomarkers mainly include effector $\mathrm{T}$ cell, $\mathrm{T}$ cell inflamed gene expression profile (GEP), tumor-infiltrating lymphocytes (TILs), and TCR sequencing. The composition of effector $\mathrm{T}$ cells, such as CD4+ $\mathrm{T}$ cell, CD8+ $\mathrm{T}$ cell, $\mathrm{T}$ regulatory cell, $\mathrm{T}$ cell inflamed GEP, TILs composition, and TCR repertoire diversity, are closely related to the efficacy of anti-PD-1/PD-L1 immunotherapy $(66,78,80)$. At present, most studies have shown that higher CD8+ T cell infiltration and lower $\mathrm{T}$ regulatory cell infiltration in tumor tissues indicate a better anti-PD-1/PD-L1 immunotherapy efficacy and better prognosis (81). The study by Han J et al. showed that TCR diversity and clonality in PD-1+ and CD8+ T cells of peripheral blood could be used as predictive biomarkers for the efficacy of anti-PD-1/PD-L1 immunotherapy (82).

\section{CONCLUSION}

Immunotherapy is a vital tumor therapeutic method, which is widely used in the treatment of NSCLC. However, the immune system is one of the most complex systems in the human body, and the related mechanisms of immunotherapy are also very complex. Specific immunotherapy efficacy and drug resistancerelated mechanisms still need a long time to explore. The most urgent problem is finding relatively stable predictive biomarkers for immunotherapy efficacy and screening out patients suitable for immunotherapy. It is believed that with the development of immunotherapy and biotechnology, immunotherapy will open up a new era of tumor therapy. 


\section{AUTHOR CONTRIBUTIONS}

FB and SG designed this research. SG, HT, NS, RZ, and MZ collected articles. FB, PS, LL, YP, GB, and BZ wrote and polished the article. All of the authors reviewed the manuscript. All authors contributed to the article and approved the submitted version.

\section{REFERENCES}

1. Siegel RL, Miller KD, Fuchs HE, Jemal A. Cancer Statistics, 2021. CA: Cancer J Clin (2021) 71(1):7-33. doi: 10.3322/caac.21654

2. Sung H, Ferlay J, Siegel RL, Laversanne M, Soerjomataram I, Jemal A, et al. Global Cancer Statistics 2020: GLOBOCAN Estimates of Incidence and Mortality Worldwide for 36 Cancers in 185 Countries. CA: Cancer J Clin (2021) 71(3):209-49. doi: 10.3322/caac.21660

3. Reck M, Carbone DP, Garassino M, Barlesi F. Targeting KRAS in Non-Small Cell Lung Cancer: Recent Progress and New Approaches. Ann Oncol: Off J Eur Soc Med Oncol (2021) 32(9):1101-10. doi: 10.1016/j.annonc.2021.06.001

4. Duma N, Santana-Davila R, Molina JR. Non-Small Cell Lung Cancer: Epidemiology, Screening, Diagnosis, and Treatment. Mayo Clin Proc (2019) 94(8):1623-40. doi: 10.1016/j.mayocp.2019.01.013

5. Andruska N, Stowe HB, Crockett C, Liu W, Palma D, Faivre-Finn C, et al. Stereotactic Radiation for Lung Cancer: A Practical Approach to Challenging Scenarios. J Thorac Oncol: Off Publ Int Assoc Study Lung Cancer (2021) 16 (7):1075-85. doi: 10.1016/j.jtho.2021.04.002

6. Pulendran B, Davis MM. The Science and Medicine of Human Immunology. Science (New York NY) (2020) 369(6511):eaay4014. doi: 10.1126/science. aay4014

7. Kim JM, Chen DS. Immune Escape to PD-L1/PD-1 Blockade: Seven Steps to Success (or Failure). Ann Oncol: Off J Eur Soc Med Oncol (2016) 27(8):1492504. doi: 10.1093/annonc/mdw217

8. Yi M, Jiao D, Xu H, Liu Q, Zhao W, Han X, et al. Biomarkers for Predicting Efficacy of PD-1/PD-L1 Inhibitors. Mol Cancer (2018) 17(1):129. doi: 10.1186/s12943-018-0864-3

9. Chen D, Barsoumian HB, Yang L, Younes AI, Verma V, Hu Y, et al. SHP-2 and PD-L1 Inhibition Combined With Radiotherapy Enhances Systemic Antitumor Effects in an Anti-PD-1-Resistant Model of Non-Small Cell Lung Cancer. Cancer Immunol Res (2020) 8(7):883-94. doi: 10.1158/23266066.CIR-19-0744

10. O’Donnell JS, Massi D, Teng MWL, Mandala M. PI3K-AKT-mTOR Inhibition in Cancer Immunotherapy, Redux. Semin Cancer Biol (2018) 48:91-103. doi: 10.1016/j.semcancer.2017.04.015

11. Jackson CM, Choi J, Lim M. Mechanisms of Immunotherapy Resistance: Lessons From Glioblastoma. Nat Immunol (2019) 20(9):1100-9. doi: 10.1038/ s41590-019-0433-y

12. O’Donnell JS, Teng MWL, Smyth MJ. Cancer Immunoediting and Resistance to T Cell-Based Immunotherapy. Nat Rev Clin Oncol (2019) 16(3):151-67. doi: 10.1038/s41571-018-0142-8

13. Sun C, Mezzadra R, Schumacher TN. Regulation and Function of the PD-L1 Checkpoint. Immunity (2018) 48(3):434-52. doi: 10.1016/j.immuni.2018.03.014

14. Chan TA, Yarchoan M, Jaffee E, Swanton C, Quezada SA, Stenzinger A, et al. Development of Tumor Mutation Burden as an Immunotherapy Biomarker: Utility for the Oncology Clinic. Ann Oncol: Off J Eur Soc Med Oncol (2019) 30 (1):44-56. doi: 10.1093/annonc/mdy495

15. Cilona M, Locatello LG, Novelli L, Gallo O. The Mismatch Repair System (MMR) in Head and Neck Carcinogenesis and Its Role in Modulating the Response to Immunotherapy: A Critical Review. Cancers (Basel) (2020) 12 (10):3006. doi: 10.3390/cancers 12103006

16. Sunshine J, Taube JM. PD-1/PD-L1 Inhibitors. Curr Opin Pharmacol (2015) 23:32-8. doi: 10.1016/j.coph.2015.05.011

17. Ramalingam S, Belani CP. State-Of-the-Art Chemotherapy for Advanced Non-Small Cell Lung Cancer. Semin Oncol (2004) 31(1 Suppl 1):68-74. doi: 10.1053/j.seminoncol.2003.12.017

18. Kalbasi A, Ribas A. Tumour-Intrinsic Resistance to Immune Checkpoint Blockade. Nat Rev Immunol (2020) 20(1):25-39. doi: 10.1038/s41577-0190218-4

\section{FUNDING}

This work was supported by the Institutional Fundamental Research Funds (2018PT32033) and the Ministry of Education Innovation Team Development Project (IRT-17R10).

19. Topalian SL, Taube JM, Anders RA, Pardoll DM. Mechanism-Driven Biomarkers to Guide Immune Checkpoint Blockade in Cancer Therapy. Nat Rev Cancer (2016) 16(5):275-87. doi: 10.1038/nrc.2016.36

20. Meyers DE, Bryan PM, Banerji S, Morris DG. Targeting the PD-1/PD-L1 Axis for the Treatment of Non-Small-Cell Lung Cancer. Curr Oncol (Toronto Ont) (2018) 25(4):e324-34. doi: 10.3747/co.25.3976

21. Ceeraz S, Nowak EC, Noelle RJ. B7 Family Checkpoint Regulators in Immune Regulation and Disease. Trends Immunol (2013) 34(11):556-63. doi: 10.1016/ j.it.2013.07.003

22. Ishida Y, Agata Y, Shibahara K, Honjo T. Induced Expression of PD-1, A Novel Member of the Immunoglobulin Gene Superfamily, Upon Programmed Cell Death. EMBO J (1992) 11(11):3887-95. doi: 10.1002/ j.1460-2075.1992.tb05481.x

23. Fife BT, Bluestone JA. Control of Peripheral T-Cell Tolerance and Autoimmunity via the CTLA-4 and PD-1 Pathways. Immunol Rev (2008) 224:166-82. doi: 10.1111/j.1600-065X.2008.00662.x

24. Liang SC, Latchman YE, Buhlmann JE, Tomczak MF, Horwitz BH, Freeman GJ, et al. Regulation of PD-1, PD-L1, and PD-L2 Expression During Normal and Autoimmune Responses. Eur J Immunol (2003) 33(10):2706-16. doi: 10.1002/eji.200324228

25. Li X, Shao C, Shi Y, Han W. Lessons Learned From the Blockade of Immune Checkpoints in Cancer Immunotherapy. J Hematol Oncol (2018) 11(1):31. doi: 10.1186/s13045-018-0578-4

26. Keir ME, Butte MJ, Freeman GJ, Sharpe AH. PD-1 and Its Ligands in Tolerance and Immunity. Annu Rev Immunol (2008) 26:677-704. doi: 10.1146/annurev.immunol.26.021607.090331

27. Yu H, Boyle TA, Zhou C, Rimm DL, Hirsch FR. PD-L1 Expression in Lung Cancer. J Thorac Oncol: Off Publ Int Assoc Study Lung Cancer (2016) 11 (7):964-75. doi: 10.1016/j.jtho.2016.04.014

28. Zhang J, Dang F, Ren J, Wei W. Biochemical Aspects of PD-L1 Regulation in Cancer Immunotherapy. Trends Biochem Sci (2018) 43(12):1014-32. doi: 10.1016/j.tibs.2018.09.004

29. Lamberti G, Spurr LF, Li Y, Ricciuti B, Recondo G, Umeton R, et al. Clinicopathological and Genomic Correlates of Programmed Cell Death Ligand 1 (PD-L1) Expression in Nonsquamous Non-Small-Cell Lung Cancer. Ann Oncol: Off J Eur Soc Med Oncol (2020) 31(6):807-14. doi: 10.1016/j.annonc.2020.02.017

30. Bailey SR, Maus MV. Gene Editing for Immune Cell Therapies. Nat Biotechnol (2019) 37(12):1425-34. doi: 10.1038/s41587-019-0137-8

31. Ma W, Gilligan BM, Yuan J, Li T. Current Status and Perspectives in Translational Biomarker Research for PD-1/PD-L1 Immune Checkpoint Blockade Therapy. J Hematol Oncol (2016) 9(1):47. doi: 10.1186/s13045016-0277-y

32. Wu SP, Liao RQ, Tu HY, Wang WJ, Dong ZY, Huang SM, et al. Stromal PDL1-Positive Regulatory T Cells and PD-1-Positive CD8-Positive T Cells Define the Response of Different Subsets of Non-Small Cell Lung Cancer to PD-1/ PD-L1 Blockade Immunotherapy. J Thorac Oncol: Off Publ Int Assoc Study Lung Cancer (2018) 13(4):521-32. doi: 10.1016/j.jtho.2017.11.132

33. Huang MY, Jiang XM, Wang BL, Sun Y, Lu JJ. Combination Therapy With PD-1/PD-L1 Blockade in Non-Small Cell Lung Cancer: Strategies and Mechanisms. Pharmacol Ther (2021) 219:107694. doi: 10.1016/ j.pharmthera.2020.107694

34. Li J, Jie HB, Lei Y, Gildener-Leapman N, Trivedi S, Green T, et al. PD-1/SHP-2 Inhibits Tc1/Th1 Phenotypic Responses and the Activation of T Cells in the Tumor Microenvironment. Cancer Res (2015) 75(3):508-18. doi: 10.1158/ 0008-5472.CAN-14-1215

35. Sacher AG, Gandhi L. Biomarkers for the Clinical Use of PD-1/PD-L1 Inhibitors in Non-Small-Cell Lung Cancer: A Review. JAMA Oncol (2016) 2 (9):1217-22. doi: 10.1001/jamaoncol.2016.0639 
36. Gong J, Chehrazi-Raffle A, Reddi S, Salgia R. Development of PD-1 and PDL1 Inhibitors as a Form of Cancer Immunotherapy: A Comprehensive Review of Registration Trials and Future Considerations. J Immunother Cancer (2018) 6(1):8. doi: 10.1186/s40425-018-0316-z

37. Chandran SS, Klebanoff CA. T Cell Receptor-Based Cancer Immunotherapy: Emerging Efficacy and Pathways of Resistance. Immunol Rev (2019) 290 (1):127-47. doi: 10.1111/imr.12772

38. Zhuang Y, Liu C, Liu J, Li G. Resistance Mechanism of PD-1/PD-L1 Blockade in the Cancer-Immunity Cycle. Onco Targets Ther (2020) 13:83-94. doi: 10.2147/OTT.S239398

39. Ramapriyan R, Caetano MS, Barsoumian HB, Mafra ACP, Zambalde EP, Menon H, et al. Altered Cancer Metabolism in Mechanisms of Immunotherapy Resistance. Pharmacol Ther (2019) 195:162-71. doi: 10.1016/j.pharmthera.2018.11.004

40. Saleh R, Elkord E. Acquired Resistance to Cancer Immunotherapy: Role of Tumor-Mediated Immunosuppression. Semin Cancer Biol (2020) 65:13-27. doi: 10.1016/j.semcancer.2019.07.017

41. Schultz L, Gardner R. Mechanisms of and Approaches to Overcoming Resistance to Immunotherapy. Hematol Am Soc Hematol Educ Program (2019) 2019(1):226-32. doi: 10.1182/hematology.2019000018

42. Li J, Stanger BZ. How Tumor Cell Dedifferentiation Drives Immune Evasion and Resistance to Immunotherapy. Cancer Res (2020) 80(19):4037-41. doi: 10.1158/0008-5472.CAN-20-1420

43. Schoenfeld AJ, Hellmann MD. Acquired Resistance to Immune Checkpoint Inhibitors. Cancer Cell (2020) 37(4):443-55. doi: 10.1016/j.ccell.2020.03.017

44. Vokes EE, Ready N, Felip E, Horn L, Burgio MA, Antonia SJ, et al. Nivolumab Versus Docetaxel in Previously Treated Advanced Non-Small-Cell Lung Cancer (CheckMate 017 and CheckMate 057): 3-Year Update and Outcomes in Patients With Liver Metastases. Ann Oncol: Off J Eur Soc Med Oncol (2018) 29(4):959-65. doi: 10.1093/annonc/mdy041

45. Rittmeyer A, Barlesi F, Waterkamp D, Park K, Ciardiello F, von Pawel J, et al. Atezolizumab Versus Docetaxel in Patients With Previously Treated NonSmall-Cell Lung Cancer (OAK): A Phase 3, Open-Label, Multicentre Randomised Controlled Trial. Lancet (London England) (2017) 389 (10066):255-65. doi: 10.1016/S0140-6736(16)32517-X

46. Patel SP, Kurzrock R. PD-L1 Expression as a Predictive Biomarker in Cancer Immunotherapy. Mol Cancer Ther (2015) 14(4):847-56. doi: 10.1158/15357163.MCT-14-0983

47. Carbone DP, Reck M, Paz-Ares L, Creelan B, Horn L, Steins M, et al. FirstLine Nivolumab in Stage IV or Recurrent Non-Small-Cell Lung Cancer. N Engl J Med (2017) 376(25):2415-26. doi: 10.1056/NEJMoa1613493

48. Hellmann MD, Ciuleanu TE, Pluzanski A, Lee JS, Otterson GA, AudigierValette C, et al. Nivolumab Plus Ipilimumab in Lung Cancer With a High Tumor Mutational Burden. N Engl J Med (2018) 378(22):2093-104. doi: 10.1056/NEJMoa1801946

49. Rizvi NA, Hellmann MD, Snyder A, Kvistborg P, Makarov V, Havel JJ, et al. Cancer Immunology. Mutational Landscape Determines Sensitivity to PD-1 Blockade in Non-Small Cell Lung Cancer. Science (New York NY) (2015) 348 (6230):124-8. doi: 10.1126/science.aaa1348

50. Gandara DR, Paul SM, Kowanetz M, Schleifman E, Zou W, Li Y, et al. BloodBased Tumor Mutational Burden as a Predictor of Clinical Benefit in NonSmall-Cell Lung Cancer Patients Treated With Atezolizumab. Nat Med (2018) 24(9):1441-8. doi: 10.1038/s41591-018-0134-3

51. Rizvi H, Sanchez-Vega F, La K, Chatila W, Jonsson P, Halpenny D, et al. Molecular Determinants of Response to Anti-Programmed Cell Death (PD)-1 and Anti-Programmed Death-Ligand 1 (PD-L1) Blockade in Patients With Non-Small-Cell Lung Cancer Profiled With Targeted Next-Generation Sequencing. J Clin Oncol: Off J Am Soc Clin Oncol (2018) 36(7):633-41. doi: 10.1200/JCO.2017.75.3384

52. Hause RJ, Pritchard CC, Shendure J, Salipante SJ. Classification and Characterization of Microsatellite Instability Across 18 Cancer Types. Nat Med (2016) 22(11):1342-50. doi: 10.1038/nm.4191

53. Umar A, Boland CR, Terdiman JP, Syngal S, de la Chapelle A, Rüschoff J, et al. Revised Bethesda Guidelines for Hereditary Nonpolyposis Colorectal Cancer (Lynch Syndrome) and Microsatellite Instability. J Natl Cancer Institute (2004) 96(4):261-8. doi: 10.1093/jnci/djh034

54. Vanderwalde A, Spetzler D, Xiao N, Gatalica Z, Marshall J. Microsatellite Instability Status Determined by Next-Generation Sequencing and Compared
With PD-L1 and Tumor Mutational Burden in 11,348 Patients. Cancer Med (2018) 7(3):746-56. doi: 10.1002/cam4.1372

55. Osmani L, Askin F, Gabrielson E, Li QK. Current WHO Guidelines and the Critical Role of Immunohistochemical Markers in the Subclassification of Non-Small Cell Lung Carcinoma (NSCLC): Moving From Targeted Therapy to Immunotherapy. Semin Cancer Biol (2018) 52(Pt 1):103-9. doi: 10.1016/ j.semcancer.2017.11.019

56. Dong ZY, Zhang JT, Liu SY, Su J, Zhang C, Xie Z, et al. EGFR Mutation Correlates With Uninflamed Phenotype and Weak Immunogenicity, Causing Impaired Response to PD-1 Blockade in Non-Small Cell Lung Cancer. Oncoimmunology (2017) 6(11):e1356145. doi: 10.1080/2162402X.2017.1356145

57. Garassino MC, Cho BC, Kim JH, Mazières J, Vansteenkiste J, Lena H, et al. Durvalumab as Third-Line or Later Treatment for Advanced Non-Small-Cell Lung Cancer (ATLANTIC): An Open-Label, Single-Arm, Phase 2 Study. Lancet Oncol (2018) 19(4):521-36. doi: 10.1016/S1470-2045(18)30144-X

58. Chen F, Zou Z, Du J, Su S, Shao J, Meng F, et al. Neoantigen Identification Strategies Enable Personalized Immunotherapy in Refractory Solid Tumors. J Clin Invest (2019) 129(5):2056-70. doi: 10.1172/JCI99538

59. Peng M, Mo Y, Wang Y, Wu P, Zhang Y, Xiong F, et al. Neoantigen Vaccine: An Emerging Tumor Immunotherapy. Mol Cancer (2019) 18(1):128. doi: 10.1186/s12943-019-1055-6

60. Wang Z, Duan J, Cai S, Han M, Dong H, Zhao J, et al. Assessment of Blood Tumor Mutational Burden as a Potential Biomarker for Immunotherapy in Patients With Non-Small Cell Lung Cancer With Use of a Next-Generation Sequencing Cancer Gene Panel. JAMA Oncol (2019) 5(5):696-702. doi: 10.1001/jamaoncol.2018.7098

61. Brozos-Vázquez EM, Díaz-Peña R, García-González J, León-Mateos L, Mondelo-Macía P, Peña-Chilet M, et al. Immunotherapy in Nonsmall-Cell Lung Cancer: Current Status and Future Prospects for Liquid Biopsy. Cancer Immunol Immunother (2021) 70(5):1177-88. doi: 10.1007/s00262-02002752-z

62. Giroux Leprieur E, Hélias-Rodzewicz Z, Takam Kamga P, Costantini A, Julie C, Corjon A, et al. Sequential ctDNA Whole-Exome Sequencing in Advanced Lung Adenocarcinoma With Initial Durable Tumor Response on Immune Checkpoint Inhibitor and Late Progression. J Immunother Cancer (2020) 8(1): e000527. doi: 10.1136/jitc-2020-000527

63. Nabet BY, Esfahani MS, Moding EJ, Hamilton EG, Chabon JJ, Rizvi H, et al. Noninvasive Early Identification of Therapeutic Benefit From Immune Checkpoint Inhibition. Cell (2020) 183(2):363-76.e13. doi: 10.1016/ j.cell.2020.09.001

64. Pitoiset F, Cassard L, El Soufi K, Boselli L, Grivel J, Roux A, et al. Deep Phenotyping of Immune Cell Populations by Optimized and Standardized Flow Cytometry Analyses. Cytometry Part A: J Int Soc Anal Cytol (2018) 93 (8):793-802. doi: 10.1002/cyto.a.23570

65. Raskov H, Orhan A, Christensen JP, Gögenur I. Cytotoxic CD8(+) T Cells in Cancer and Cancer Immunotherapy. Br J Cancer (2021) 124(2):359-67. doi: 10.1038/s41416-020-01048-4

66. He Q, Liu Z, Liu Z, Lai Y, Zhou X, Weng J. TCR-Like Antibodies in Cancer Immunotherapy. J Hematol Oncol (2019) 12(1):99. doi: 10.1186/s13045-0190788-4

67. Fumet JD, Richard C, Ledys F, Klopfenstein Q, Joubert P, Routy B, et al. Prognostic and Predictive Role of CD8 and PD-L1 Determination in Lung Tumor Tissue of Patients Under Anti-PD-1 Therapy. Br J Cancer (2018) 119 (8):950-60. doi: 10.1038/s41416-018-0220-9

68. Gettinger SN, Choi J, Mani N, Sanmamed MF, Datar I, Sowell R, et al. A Dormant TIL Phenotype Defines Non-Small Cell Lung Carcinomas Sensitive to Immune Checkpoint Blockers. Nat Commun (2018) 9(1):3196. doi: 10.1038/s41467-018-05032-8

69. Anagnostou V, Forde PM, White JR, Niknafs N, Hruban C, Naidoo J, et al. Dynamics of Tumor and Immune Responses During Immune Checkpoint Blockade in Non-Small Cell Lung Cancer. Cancer Res (2019) 79(6):1214-25. doi: 10.1158/0008-5472.CAN-18-1127

70. Xie F, Xu M, Lu J, Mao L, Wang S. The Role of Exosomal PD-L1 in Tumor Progression and Immunotherapy. Mol Cancer (2019) 18(1):146. doi: 10.1186/ s12943-019-1074-3

71. An HJ, Chon HJ, Kim C. Peripheral Blood-Based Biomarkers for Immune Checkpoint Inhibitors. Int J Mol Sci (2021) 22(17):9414. doi: 10.3390/ ijms22179414 
72. Tucci M, Passarelli A, Mannavola F, Stucci LS, Ascierto PA, Capone M, et al. Serum Exosomes as Predictors of Clinical Response to Ipilimumab in Metastatic Melanoma. Oncoimmunology (2018) 7(2):e1387706. doi: 10.1080/ 2162402X.2017.1387706

73. Showalter A, Limaye A, Oyer JL, Igarashi R, Kittipatarin C, Copik AJ, et al. Cytokines in Immunogenic Cell Death: Applications for Cancer Immunotherapy. Cytokine (2017) 97:123-32. doi: 10.1016/j.cyto.2017.05.024

74. Vétizou M, Pitt JM, Daillère R, Lepage P, Waldschmitt N, Flament C, et al. Anticancer Immunotherapy by CTLA-4 Blockade Relies on the Gut Microbiota. Science (New York NY) (2015) 350(6264):1079-84. doi: $10.1126 /$ science.aad1329

75. Elkrief A, Derosa L, Zitvogel L, Kroemer G, Routy B. The Intimate Relationship Between Gut Microbiota and Cancer Immunotherapy. Gut Microbes (2019) 10(3):424-8. doi: 10.1080/19490976.2018.1527167

76. Marin-Acevedo JA, Soyano AE, Dholaria B, Knutson KL, Lou Y. Cancer Immunotherapy Beyond Immune Checkpoint Inhibitors. J Hematol Oncol (2018) 11(1):8. doi: 10.1186/s13045-017-0552-6

77. Chauvin JM, Zarour HM. TIGIT in Cancer Immunotherapy. J Immunother Cancer (2020) 8(2):e000957. doi: 10.1136/jitc-2020-000957

78. Paijens ST, Vledder A, de Bruyn M, Nijman HW. Tumor-Infiltrating Lymphocytes in the Immunotherapy Era. Cell Mol Immunol (2021) 18 (4):842-59. doi: 10.1038/s41423-020-00565-9

79. Berraondo P, Sanmamed MF, Ochoa MC, Etxeberria I, Aznar MA, PérezGracia JL, et al. Cytokines in Clinical Cancer Immunotherapy. Br J Cancer (2019) 120(1):6-15. doi: 10.1038/s41416-018-0328-y

80. Cristescu R, Mogg R, Ayers M, Albright A, Murphy E, Yearley J, et al. PanTumor Genomic Biomarkers for PD-1 Checkpoint Blockade-Based
Immunotherapy. Science (New York NY) (2018) 362(6411):eaar3593. doi: 10.1126/science.aar3593

81. Nelson MA, Ngamcherdtrakul W, Luoh SW, Yantasee W. Prognostic and Therapeutic Role of Tumor-Infiltrating Lymphocyte Subtypes in Breast Cancer. Cancer Metastasis Rev (2021) 40(2):519-36. doi: 10.1007/s10555-021-09968-0

82. Han J, Duan J, Bai H, Wang Y, Wan R, Wang X, et al. TCR Repertoire Diversity of Peripheral PD-1(+)CD8(+) T Cells Predicts Clinical Outcomes After Immunotherapy in Patients With Non-Small Cell Lung Cancer. Cancer Immunol Res (2020) 8(1):146-54. doi: 10.1158/2326-6066.CIR-19-0398

Conflict of Interest: The authors declare that the research was conducted in the absence of any commercial or financial relationships that could be construed as a potential conflict of interest.

Publisher's Note: All claims expressed in this article are solely those of the authors and do not necessarily represent those of their affiliated organizations, or those of the publisher, the editors and the reviewers. Any product that may be evaluated in this article, or claim that may be made by its manufacturer, is not guaranteed or endorsed by the publisher.

Copyright (c) 2022 Bie, Tian, Sun, Zang, Zhang, Song, Liu, Peng, Bai, Zhou and Gao. This is an open-access article distributed under the terms of the Creative Commons Attribution License (CC BY). The use, distribution or reproduction in other forums is permitted, provided the original author(s) and the copyright owner(s) are credited and that the original publication in this journal is cited, in accordance with accepted academic practice. No use, distribution or reproduction is permitted which does not comply with these terms. 